\title{
A Reflector Antenna Concept Robust Against Feed Failures for Satellite Communications
}

\author{
Sigurd Huber, Marwan Younis, Senior Member, IEEE, Gerhard Krieger, Fellow, IEEE, \\ Alberto Moreira, Fellow, IEEE, and Werner Wiesbeck, Fellow, IEEE
}

\begin{abstract}
Since the first communications satellites have been launched to space with the beginning of the 1960s, these systems have undergone a rapid development. Amongst others, this development is driven by an increasing number of subscribers exchanging larger and larger data volumes. This need of data capacity cannot be satisfied alone by raising the sheer number of communications satellites, but requires powerful individual systems, which operate reliably and are cost effective at the same time. In this context two requirements on the communications antenna are the provision of high directional gain and robustness in terms of beam stability. Classically, large unfurlable mesh reflector antennas in conjunction with feed arrays are adopted to illuminate a certain region on ground with high gain. An inherent problem of such reflector-feed configurations is that these systems are prone to feed element failures. In the worst case, this could result in a 'blind' spot, where no communication is possible. This paper introduces a robust antenna concept, which combines the virtue of reflector antennas, namely the large aperture, with the advantage of direct radiating planar array antennas, which is the beam stability in the presence of element failures. In order to unfold its full potential this concept makes use of digital beamforming techniques, which allow to control the illumination in a flexible way.
\end{abstract}

Index Terms-digital beamforming, DBF, multiple-input multiple-output, MIMO, defocused, reflector antennas, satellite communications

\section{INTRODUCTION}

A NTENNA concepts with application to communications satellites have been studied extensively in the past years. In this context large unfoldable mesh reflector antennas represent a mature technology, which is employed on many communications systems. In conjunction with feed arrays these reflectors are able to illuminate communication cells on the Earth surface with high gain. Consequently, a lot of effort has been put in the optimization of feed antennas [1] as well as entire reflector-feed configurations [2]-[5].

Already in the 1990s the idea of beamforming has been investigated for communications systems [6]. However, beamforming has been carried out by means of analog networks. A few years later the concept of digital beamforming (DBF) found one's way into satellite communications [7].

This paper attempts to bring array-fed reflector antennas in symbiosis with digital beamforming in a different way. The innovation lies in the fact that the reflector is intentionally

Manuscript received April 30, 2014. S. Huber, G. Krieger and A. Moreira are with the Microwaves and Radar Institute, German Aerospace Center (DLR), Oberpfaffenhofen, Germany, e-mail: sigurd.huber@dlr.de.

W. Wiesbeck is with the Institute of High Frequency Techniques and Electronics (IHE), Karlsruhe Institute of Technology (KIT), Karlsruhe, Germany

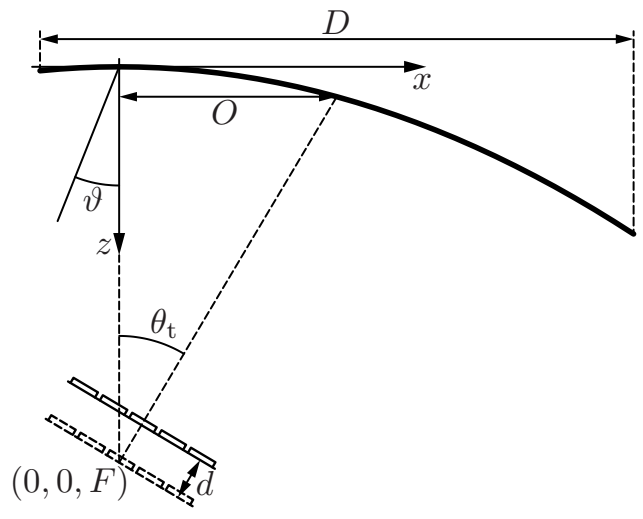

Fig. 1: Cut view of a reflector with center-offset fed by a two-dimensional planar array. The reflector is defocused by introducing a linear shift of the feed array.

defocused and therefore relies on DBF techniques in order to be operated efficiently. The main goal of this concept is to improve the robustness of such systems in the presence of feed element failures, which also enables to efficiently compensate for feed element deterioration. This allows to abstain from redundant electronics and therefore reduces costs.

\section{Defocused Reflector Antennas}

The basic concept of a (single) reflector antenna is to transform a primary field, incident on the reflector, into a secondary field, which is usually referred to as the field or the pattern of the array-fed reflector antenna. The source of the primary field is typically a feed antenna or an array of feed antennas. A widely established reflector type is the parabolic reflector, whose surface may be described in cartesian coordinates according to

$$
z=\frac{1}{4 F}\left(x^{2}+y^{2}\right)
$$

As schematically indicated in Fig. $1 F$ is the distance between the apex of the reflector and the focal point $(0,0, F)$ given in local antenna coordinates $(x, y, z)$. Characteristic for such reflectors is that they concentrate the field of an incident plane wave in a small region. If the plane wave impinges the reflector in negative $z$-direction the point of highest field strength is the focal point $(0,0, F)$, short focus. In order to illuminate a large angular domain several feed elements are arranged in the focal plane, each illuminating a distinct essentially non-overlapping solid angle. 


\begin{tabular}{l|c|c} 
parameter & symbol & value \\
\hline frequency & $f$ & $1.25 \mathrm{GHz}$ \\
diameter & $D$ & $15 \mathrm{~m}$ \\
focal length & $F$ & $10 \mathrm{~m}$ \\
center-offset & $O$ & $5.5 \mathrm{~m}$ \\
feed array shift & $d$ & $0.55 \mathrm{~m}$ \\
feed element spacing & & $1.0 \lambda$ \\
number of feed elements & $N$ & $9 \times 9$
\end{tabular}

TABLE I: Geometrical parameters for a reflector design example at an L-band frequency. The feed element spacing is identical for both dimensions of the two-dimensional feed array.

Defocused reflector antennas have been suggested for instance in [8]-[10], partly out of field-theoretical interests but also as a method to shape the pattern. In the context of this paper the term 'defocused' shall be understood in a quite general way, meaning that adjacent feed elements may have a substantial overlap in their corresponding patterns. Insofar, direct radiating arrays can be regarded as a limit of this defocused antenna concept, where all element patterns perfectly coincide. Of course, this state can never be reached with reflector based antennas. However, a certain degree of defocusing would certainly improve the robustness in terms of pattern shape stability in case of feed element failures.

To demonstrate this concept consider the cut view of a parabolic reflector in Fig. 1. The center-offset $O$ has been introduced in order to mitigate multipath effects occurring between the feed array and the reflector. This requires the feed array being tilted by an angle

$$
\theta_{\mathrm{t}}=2 \arctan \left(\frac{O}{2 F}\right)
$$

towards the center of the projected aperture of diameter $D$. In the focused case the feed array would run through the focal point $(0,0, F)$, as indicated by the dashed feed array. Here, the defocusing is achieved by a linear shift $d$ of the feed array as sketched in Fig. 1. The question whether a shift in this or the opposite direction is preferable depends on how well the reflector can be illuminated by the feed antennas. In principle, both shift directions are reasonable, at least from an electrical point of view. With regard to cost effectiveness a design goal is to select a low number of feed elements. This results in a relatively large element spacing of $1.0 \lambda$ for $9 \times 9$ patch elements arranged in a rectangular grid. Figure 2 shows the simulation results of the nine far-field gain patterns $G_{i}$ corresponding to the individual feed elements in the center row (in the plane of Fig. 1) of the array. These patterns have been computed with the EM-simulation software TICRA GRASP10 [11], on the basis of a microstrip patch model for the feed elements as presented in [12]. The parameters for the design example are summarized in Table I. In order to determine the fields as accurately as possible also multipath propagation between the reflector and the feed array, modeled as a perfectly conducting metal plate, has been taken into account. As can be observed by comparing the patterns of the defocused system in Fig. 2a with the conventional beams in Fig. 2b, the gain loss is in the order of $6 \mathrm{~dB}$. At the same time the defocused patterns

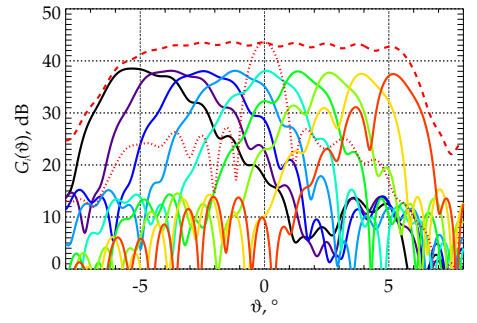

(a) defocused reflector

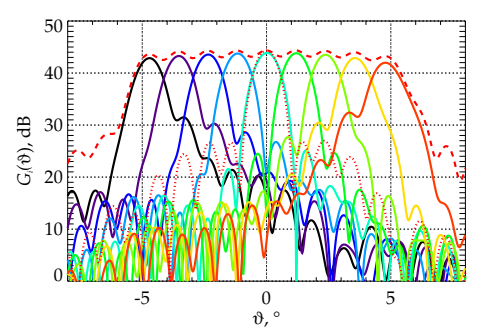

(b) conventional reflector

Fig. 2: Beam patterns in a cut view corresponding to the nine elements in the center row of the feed array for the defocused reflector (a) and for the conventional reflector (b). The dashed curves represent patterns after MVDR beamforming according to equation (13). The dotted red beams represent a MVDR beam for $\vartheta=0^{\circ}$.

show a significant broadening. Clearly, such a system could not be operated in the usual way where each beam illuminates a distinct cell with a high gain beam. This is especially important if a high co-polar isolation in case of the reuse of frequency bands is required. In the following the performance of such a defocused reflector concept shall be investigated on the basis of two selected beamforming approaches.

\section{Signal Model And DBF Techniques}

Principally, different operation modes for reflector antennas mounted on satellites must be discriminated. For instance, radio broadcasting usually requires an entire country or continent to be homogeneously illuminated by the reflector antenna. In communications typically small cells are illuminated with high gain beams. Each of these applications involves pattern optimization with certain goals and constraints. For example in satellite communications an optimization goal would be the minimization of the pattern sidelobes in the entire access domain while keeping the gain in the desired cell at a maximum. Here, the performance of the defocused reflector concept shall be demonstrated at the example of point-to-point communications adopting MIMO (multiple-input multipleoutput) principles, similar to the concept presented in [6]. Starting point for the digital beamforming techniques is a model for the received channel signals

$$
\boldsymbol{u}=\boldsymbol{A s}+\boldsymbol{v} .
$$

The array response matrix

$$
\boldsymbol{A}=\left[\begin{array}{llll}
\boldsymbol{a}\left(\vartheta_{1}, \varphi_{1}\right) & \boldsymbol{a}\left(\vartheta_{2}, \varphi_{2}\right) & \cdots & \boldsymbol{a}\left(\vartheta_{M}, \varphi_{M}\right)
\end{array}\right]
$$




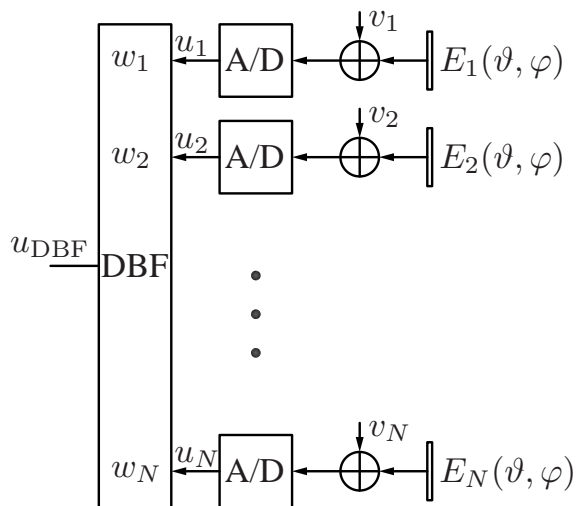

Fig. 3: System model of the digital feed array. The received signals are digitized and combined in the DBF unit.

collects $M$ complex array manifold vectors $\boldsymbol{a}$, each associated with a certain direction $(\vartheta, \varphi)$. For instance, the direction $\left(\vartheta_{1}, \varphi_{1}\right)$ could be associated with the user of interest, while the $M-1$ other directions represent other users in the same frequency band to be suppressed. The corresponding signals are combined in the vector

$$
\boldsymbol{s}=\left[\begin{array}{llll}
s_{1}\left(\vartheta_{1}, \varphi_{1}\right) & s_{2}\left(\vartheta_{2}, \varphi_{2}\right) & \cdots & s_{M}\left(\vartheta_{M}, \varphi_{M}\right)
\end{array}\right]^{\top},
$$

where $\{.\}^{\top}$ symbolizes transpose. In this context the complex array manifold vector

$$
\boldsymbol{a}(\vartheta, \varphi)=\left[\begin{array}{llll}
E_{1}(\vartheta, \varphi) & E_{2}(\vartheta, \varphi) & \cdots & E_{N}(\vartheta, \varphi)
\end{array}\right]^{\top}
$$

contains the co-polar electric far field patterns $E$, after deflection at the reflector, as function of the spherical angles $(\vartheta, \varphi)$ defined in the local antenna coordinate system. It is important to note that these complex amplitude patterns are so called embedded patterns. Each receiver channel is superimposed by thermal receiver noise $v$. This system model is graphically illustrated in Fig. 3, where the feed array with analog-to-digital (A/D) converters and digital beamforming unit is depicted. Note, in order to keep a clear representation, amplifiers, filters, mixers and other components of the receiver electronics have been omitted.

The core operation in the DBF unit is the combination of the individual received signals $u$, yielding the beamformer output

$$
u_{\mathrm{DBF}}=\boldsymbol{w}^{\top} \boldsymbol{u} .
$$

Finding meaningful weights $\boldsymbol{w}$ is a research field on its own, known as pattern synthesis problem. In this context two beamforming concepts shall be considered, which can be derived from a power expression of the beamformer output according to

$$
\begin{aligned}
P_{\mathrm{DBF}} & =\mathcal{E}\left\{\left|u_{\mathrm{DBF}}\right|^{2}\right\} \\
& =\left|\boldsymbol{w}^{\top} \boldsymbol{A} \boldsymbol{s}\right|^{2}+\boldsymbol{w}^{\top} \mathcal{E}\left\{\boldsymbol{v} \boldsymbol{v}^{\mathrm{H}}\right\} \boldsymbol{w}^{*},
\end{aligned}
$$

with $\mathcal{E}\{$.$\} denoting expectation value, \{.\}^{*}$ conjugate complex and $\{.\}^{\mathrm{H}}$ conjugate complex transpose. Here, statistically independent signal and noise contributions are assumed. The expression $\mathcal{E}\left\{\boldsymbol{v} \boldsymbol{v}^{\mathrm{H}}\right\}$ is known as noise channel covariance matrix $\boldsymbol{R}_{v}$, which might be estimated from samples of the data stream. Then an optimization problem

$$
\begin{array}{cl}
\operatorname{minimize} & \boldsymbol{w}^{\top} \boldsymbol{R}_{\boldsymbol{v}} \boldsymbol{w}^{*} \\
\text { subject to } & \boldsymbol{w}^{\top} \boldsymbol{A}=\boldsymbol{c}
\end{array}
$$

can be formulated, which has the analytic solution

$$
\boldsymbol{w}^{*}=\boldsymbol{R}_{\boldsymbol{v}}^{-1} \boldsymbol{A}\left(\boldsymbol{A}^{\mathrm{H}} \boldsymbol{R}_{v}^{-1} \boldsymbol{A}\right)^{-1} \boldsymbol{c}^{*} .
$$

The vector $\boldsymbol{c}$ is a so called constraint vector, which may be chosen almost arbitrarily, depending on the application. If one recalls the above example, $c$ would have a '1' associated to the direction of interest, and zeros in the directions to be damped. In the literature this solution is known as Linear Constraint Minimum Variance (LCMV) beamformer [13]. A special case of this beamformer is the Minimum Variance Distortionless Response (MVDR) beamformer, that is obtained for $M=1$, giving

$$
\boldsymbol{w}^{*}=\frac{c^{*} \boldsymbol{R}_{v}^{-1} \boldsymbol{a}}{\boldsymbol{a}^{\mathrm{H}} \boldsymbol{R}_{v}^{-1} \boldsymbol{a}} .
$$

MVDR beamforming can be understood as a spatial matched filter and as such optimizes the gain or equivalently the signalto-noise ratio (SNR) in the respective direction of interest. In the context of this paper, the purpose of the MVDR beamformer is to demonstrate the maximum achievable gain in a certain direction, which is of special interest under failure conditions.

The MVDR solution interpreted in terms of DBF gain is shown in Fig. 2 as dashed red curves. It is important to note, that these curves are the result when for each angle $\vartheta$ a beam is formed. This means for each angle $\vartheta$ the individual patterns (81 in our case) are combined on the basis of the MVDR weights given in equation (13). Just for illustration purposes a single MVDR beam for $\vartheta=0^{\circ}$ has been plotted. Comparing the dashed curves from Figures $2 \mathrm{a}$ and $2 \mathrm{~b}$ it becomes evident that in case of the defocused antenna the high gain is recovered by means of digital beamforming almost as well as in the reference case. A slight degradation in gain, especially at the borders of the illuminated domain, is inevitable, since the defocused reflector radiates a certain amount of energy beyond the access range of $\pm 5^{\circ}$.

An important question is whether the defocusing handicaps the antenna in terms of beam shaping. There seem to be no obvious limitations as can be seen from the two-dimensional pattern plots in Fig. 4. Here, a scenario is considered where three other users to be damped are present under the polar angles $\left(\xi=-4^{\circ}, \zeta=4^{\circ}\right),\left(2^{\circ}, 3^{\circ}\right)$ and $\left(1^{\circ},-2^{\circ}\right)$. Note, the polar angles are linked to the spherical coordinates via

$$
\begin{aligned}
& \xi=\vartheta \cos \varphi, \\
& \zeta=\vartheta \sin \varphi .
\end{aligned}
$$

The beam has been steered to the direction of interest at $\left(-2^{\circ},-2^{\circ}\right)$ using LCMV beamforming. In order to pronounce the suppressed directions, in total four zero constraints have been placed in a quadratic grid at each direction to be suppressed. This can, for example, be observed at the pattern zero at $\left(-4^{\circ}, 4^{\circ}\right)$ in Fig. $4 \mathrm{~b}$. The maximum gain with 


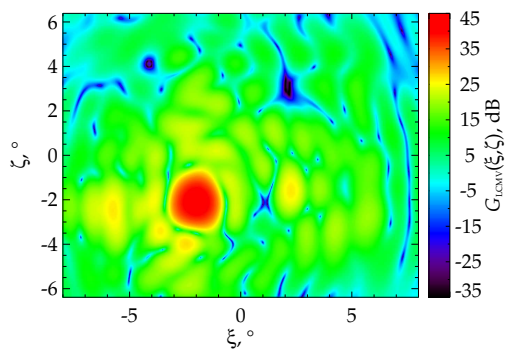

(a) defocused reflector

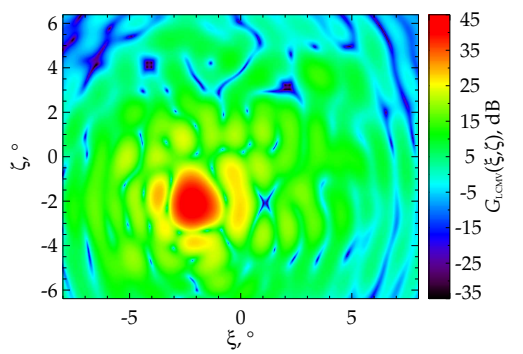

(b) conventional reflector

Fig. 4: Two-dimensional pattern plots with three suppressed directions at $\left(-4^{\circ}, 4^{\circ}\right),\left(2^{\circ}, 3^{\circ}\right)$ and $\left(1^{\circ},-2^{\circ}\right)$ using LCMV beamforming according to equation (12) for the defocused case (a) and the conventional reflector (b).

\begin{tabular}{l|c|c|c|c} 
reflector & \multicolumn{2}{|c|}{ defocused } & \multicolumn{2}{c}{ conventional } \\
\hline$\vartheta,^{\circ}$ & 0.0 & 3.56 & 0.0 & 3.56 \\
\hline$G_{\mathrm{MVDR}}(\vartheta), \mathrm{dB}$ & 43.53 & 43.07 & 44.33 & 43.98 \\
$G_{\mathrm{MVDR}, \text { fail }}(\vartheta), \mathrm{dB}$ & 42.08 & 42.04 & 34.52 & 37.51 \\
\hline$\Delta G(\vartheta), \mathrm{dB}$ & -1.46 & -1.03 & -9.82 & -6.48
\end{tabular}

TABLE II: Performance comparison after MVDR beamforming in terms of gain loss in the presence of feed failures.

the conventional reflector is $0.58 \mathrm{~dB}$ higher compared to the defocused case.

\section{Performance under Failure Conditions}

The major motivation to employ such a defocused reflector concept is its robustness when individual or even multiple feed elements drop out. Failure-critical components in this context are usually the amplifiers. Here, a scenario is investigated where the fifth and the eighth feed element in the center row of the feed array have dropped out. The analogous results to Fig. 2 are plotted in Fig. 5. Again the dashed red curve, which represents the gain pattern after MVDR beamforming, is of importance. Noticeable is that the gain drop in the center at $0^{\circ}$ is maximal, while the gain loss in the direction of the eighth element is not as large but still significant for the conventional reflector. This can be explained by the fact that in the conventional case the patterns corresponding to the feed elements at the border of the array are more strongly defocused than the patterns of the center feed elements. In contrast, the defocused reflector suffers much less from the failed elements, which can be seen from the performance comparison in Table II. Here, the gain before and after element failure for

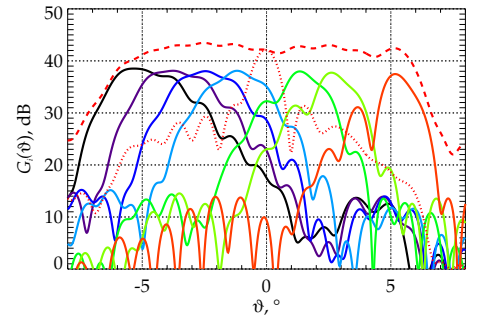

(a) defocused reflector

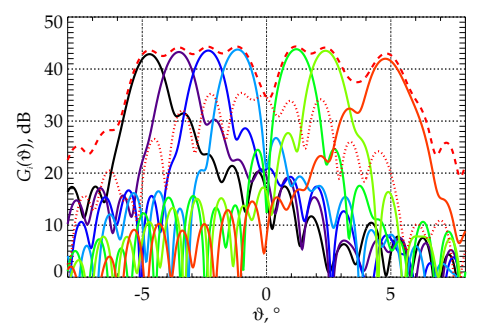

(b) conventional reflector

Fig. 5: Beam patterns in a cut view corresponding to the nine elements in the center row of the feed array for the defocused reflector (a) and for the conventional reflector (b). Here, the fifth and the eighth element have dropped out. The dashed curves represent patterns after MVDR beamforming according to equation (13). The dotted red beams represent a MVDR beam for $\vartheta=0^{\circ}$.

two dedicated directions are compared. A first observation is that the defocused reflector generally has a slightly reduced gain after MVDR beamforming, as can be seen from the row listing the gain $G_{\text {MVDR }}(\vartheta)$ for the case when all feed elements are operating (see Fig. 2). The next row shows the gain $G_{\text {MVDR,fail }}(\vartheta)$ in the directions of the two dropped out feed elements. In the last row the difference in gain $\Delta G(\vartheta)$ before and after feed element failure is presented. Here, it becomes evident that the conventional reflector antenna would be severely handicapped, without relying on redundancy concepts and if not enough margin in terms of SNR had been planned in the communications system design.

\section{Extension to Curved FeEd Array}

A certain disadvantage of defocused reflector systems is the gain roll-off towards the borders of the illuminated domain. Here basically two options might be of interest. The first possibility would simply involve additional feed elements at

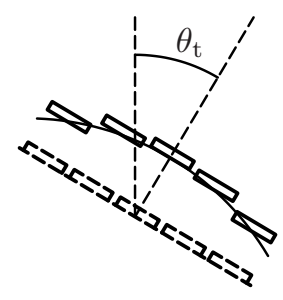

Fig. 6: Curved feed array design.

the borders of the array in order to compensate the gain loss. 
A second option could be the use of a curved feed array as sketched in Fig. 6. Here, again in dashed lines the feed

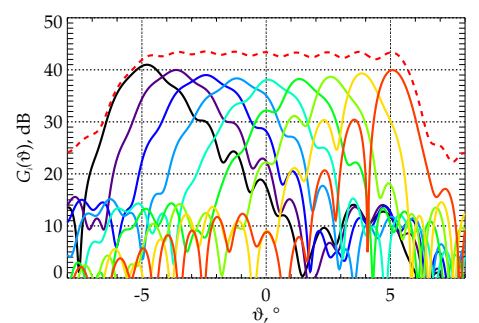

(a) defocused reflector

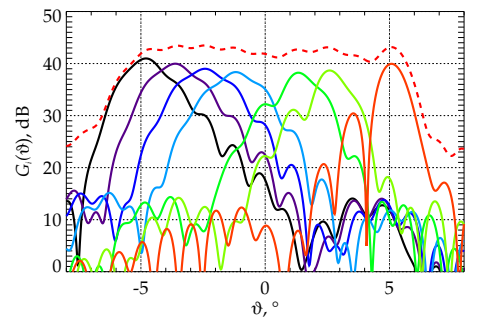

(b) defocused reflector with dropouts

Fig. 7: Beam patterns in a cut view corresponding to the nine elements in the center row of the curved feed array for the defocused reflector without element failures (a) and with elements five and eight dropped out (b). The dashed curves represent patterns after MVDR beamforming according to equation (13).

array of the conventional reflector is drawn. The 2-D feed array is bent such that the outer elements come closer to the position of the outer feed elements of the conventional feed array. Consequently, these elements will show a reduced defocusing, as can be observed in the pattern cut plot in Fig. 7a. In case of feed element failures this reflector-feed concept shows a similar but slightly worse performance as the defocused reflector in Fig. 5a. Assuming the same failure scenario, the gain loss is $-1.52 \mathrm{~dB}$ for zero degree direction and $-1.70 \mathrm{~dB}$ at $3.56^{\circ}$ (see Fig. $7 \mathrm{~b}$ ). Of course, the gain loss in case of border element failures is now slightly increased but still much better as in the conventional case. A more general approach could be the optimization of the reflector surface together with the feed array.

\section{CONCLUSION}

This paper presents a study of a defocused reflector-feed system concept employing digital beamforming techniques for communication satellites. The defocusing has been achieved by shifting the 2-D feed array away from the focal plane towards the reflector. As a consequence the patterns associated with the individual feed elements become broader at simultaneously reduced maximum gain. By applying digital beamforming techniques the high gain could be reconstructed without any limitations. This concept combines the advantage of reflector antennas, which is the large realizable aperture, with the robustness of direct radiating arrays in the presence of feed failures.

\section{REFERENCES}

[1] E. G. Geterud, J. Yang, T. Ostling, and P. Bergmark, "Design and Optimization of a Compact Wideband Hat-Fed Reflector Antenna for Satellite Communications," IEEE Transactions on Antennas and Propagation, vol. 61, no. 1, pp. 125-133, Jan 2013.

[2] R. Jorgensen, P. Balling, and W. English, "Dual Offset Reflector Multibeam Antenna for International Communications Satellite Applications," IEEE Transactions on Antennas and Propagation, vol. 33, no. 12, pp. 1304-1312, Dec 1985.

[3] S. K. Rao, "Parametric Design and Analysis of Multiple-Beam Reflector Antennas for Satellite Communications," IEEE Antennas and Propagation Magazine, vol. 45, no. 4, pp. 26-34, Aug 2003.

[4] S. K. Rao and M. Q. Tang, "Stepped-Reflector Antenna for Dual-Band Multiple Beam Satellite Communications Payloads," IEEE Transactions on Antennas and Propagation, vol. 54, no. 3, pp. 801-811, Mar 2006.

[5] P.-S. Kildal, "Some Reflector and Feed Antenna Inventions that Made a Difference: Fundamentals and examples from radio telescopes, satellite communications and radio links," in AFRICON, Sep 2013, pp. 1-3.

[6] T. Gebauer and H. G. Gockler, "Channel-Individual Adaptive Beamforming for Mobile Satellite Communications," IEEE Journal on Selected Areas in Communications, vol. 13, no. 2, pp. 439-448, Feb 1995.

[7] W. Lo and J. Chang, "Digital Beamforming in a Satellite Communication System," U.S. Patent 6,016,124, Jan, 2000.

[8] D. K. Cheng and S. T. Moseley, "On-Axis Defocus Characteristics of the Paraboloidal Reflector," IRE Transactions on Antennas and Propagation, pp. 214-216, Oct 1955.

[9] R. Yang, "Illuminating curved passive reflector with defocused parabolic antenna," in WESCON/58 Conference Record, vol. 2, Aug 1958, pp. 260-265.

[10] A. D. Olver and J. U. I. Syed, "Variable beamwidth reflector antenna by feed defocusing," IEE Proceedings - Microwaves, Antennas and Propagation, vol. 142, no. 5, pp. 394-398, Oct 1995.

[11] "General reflector and antenna farm analysis software," http://www.ticra.com, TICRA, Aug 2011.

[12] L. Lei, G. Zhang, and R. J. Doviak, "Bias Correction for Polarimetric Phased-Array Radar With Idealized Aperture and Patch Antenna Elements," IEEE Transactions on Geoscience and Remote Sensing, vol. 51, no. 1, pp. 473-486, Jan 2013.

[13] H. L. V. Trees, Optimum Array Processing. John Wiley \& Sons, Inc., 2002.

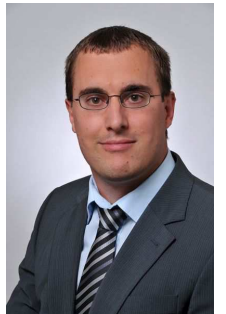

Sigurd Huber received the Dipl.-Ing. (M.S.) and Dr.-Ing. (Ph.D.) (with summa honors) degrees in electrical and communication engineering from the Technical University of Munich (TUM), Germany, in 2005 and the Karlsruhe Institute of Technology (KIT), Germany, in 2014, respectively.

Since 2005 he is with the Microwaves and Radar Institute of the German Aerospace Center (DLR), Oberpfaffenhofen, Germany, where he is engaged in the conception of future synthetic aperture radar systems, utilizing advanced multichannel antenna architectures. He is involved in national and international projects in the field of Earth observation, encompassing cooperations with industry partners (Airbus), as well as research organizations (ESA, NASA/JPL and JAXA). He contributed as author and co-author in more than 35 conference papers and five peer reviewed journal articles. His research interests include digital signal processing, electromagnetic field theory, inverse problems, array processing and antenna theory. 


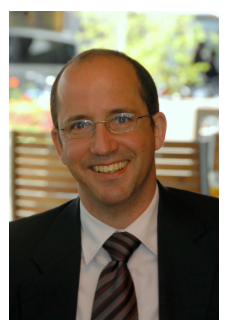

Marwan Younis (S'1995, M'2005, SM'2008) received his B.Sc in electrical engineering from the University of Baghdad, Iraq in 1992 and the Dipl.Ing. (M.Sc.) and Dr.-Ing. (Ph.D.) degree in electrical engineering from the Universität Karlsruhe (TH), Germany, in 1997 and 2004, respectively.

From 1998 to 2004, he was a research scientist with the Institut für Höchstfrequenztechnik und Elektronik, Universität Karlsruhe (TH). Since 2005 he has been with the Microwaves and Radar Institute of the German Aerospace Center (DLR), Oberpfaffenhofen, Germany. He is the author and co-author of about 120 conference papers and 25 reviewed publications. His research fields include synthetic aperture radar (SAR) systems and techniques, MIMO SAR, digital beamforming, SAR performance, calibration, and antennas. In 1996 he was an intern at the Jet Propulsion Laboratory (JPL) and in 2013 he spent a three month research sabbatical at JPL.

Dr. Younis is an active member of the IEEE. He co-chairs the Instrumentation and Future Technologies GRSS Technical Committee. He is associate editor for the IEEE geoscience and remote sensing letter and reviewer of IEEE publications. He is a lecturer with the Universität Karlsruhe (TH) and the Carl-Cranz-Gesellschaft. He received the Hermann-Billing award for his Ph.D. thesis in 2005 .

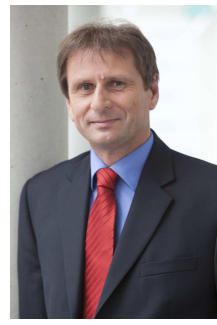

Gerhard Krieger received the Dipl.-Ing. (M.S.) and Dr.-Ing. (Ph.D.) degrees (with honors) in electrical and communication engineering from the Technical University of Munich, Germany, in 1992 and 1999, respectively.

From 1992 to 1999, he was with the Ludwig Maximilians University, Munich, where he conducted multidisciplinary research on neuronal modeling and nonlinear information processing in biological and technical vision systems. Since 1999, he has been with the Microwaves and Radar Institute (HR) of the German Aerospace Center (DLR), Oberpfaffenhofen, Germany, where he developed signal and image processing algorithms for a novel forward looking radar system employing digital beamforming on receive. From 2001 to 2007 he led the New Synthetic Aperture Radar (SAR) Missions Group which pioneered the development of advanced bistatic and multistatic radar systems as exemplified by the TanDEM-X mission, as well as innovative multichannel SAR techniques and algorithms for high-resolution wide-swath SAR imaging. Since 2008, he has been the Head of the new Radar Concepts Department of the Microwaves and Radar Institute, DLR, Oberpfaffenhofen, Germany. Gerhard Krieger is a visiting lecturer at the Friedrich Alexander University, Erlangen, Germany, and has authored/co-authored 65 peer-reviewed journal papers, 7 invited book chapters, about 300 conference papers, and more than 10 patents. His current research interests focus on the development of multichannel radar techniques and algorithms for innovative MIMO and cognitive SAR systems, wave propagation effects in the tropo- and ionosphere, the demonstration of novel interferometric and tomographic Earth observation applications, and the conceptual design of advanced bi- and multistatic radar missions.

Dr. Krieger is IEEE Fellow and received several national and international awards, including the W.R.G. Baker Prize Paper Award from the IEEE Board of Directors and the Transactions Prize Paper Award of the IEEE Geoscience and Remote Sensing Society. In 2012, he and his colleagues were nominated for the German President's Award for Technology and Innovation. Since 2012, he has been Associate Editor of the IEEE Transactions on Geoscience and Remote Sensing.

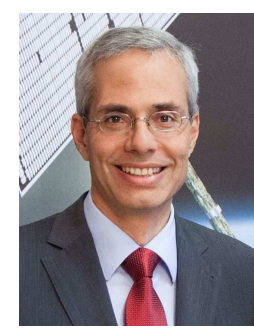

Alberto Moreira (M'92, S'96, F'04) received the B.S.E.E. and M.S.E.E. degrees from the Aeronautical Technological Institute (ITA), São José dos Campos, in 1984 and 1986, respectively, and the Eng. Dr. degree (with honors) from the Technical University of Munich, Germany, in 1993. From 1996 to 2001, he was the Chief Scientist and Engineer with the SAR Technology Department, German Aerospace Center (DLR), Oberpfaffenhofen, Germany. Under his leadership, the DLR airborne SAR system has been upgraded to operate in innovative imaging modes like polarimetric SAR interferometry and SAR tomography. Since 2001, he has been the Director of the Microwaves and Radar Institute at DLR. The Institute contributes to several scientific programs and space projects for actual and future airborne and spaceborne SAR missions like TerraSARX, TanDEM-X, PAZ, Sentinel-1, SAR-Lupe, BIOMASS and Tandem-L. The mission TanDEM-X, led by his Institute, has successfully started the operational phase in December 2010. Prof. Moreira is the initiator and Principal Investigator for this mission. Since 2003, he is also a Full Professor with the Karlsruhe Institute of Technology, Germany, in the field of microwave remote sensing. He has more than 350 publications in international conferences and journals and is the holder of 18 patents in the radar and antenna field. His professional interests and research areas encompass radar end-to-end system design and analysis, microwave technologies, techniques and systems, signal and image processing, and remote sensing applications.

He served as President of the IEEE Geoscience and Remote Sensing Society (GRSS) in 2010, was the Founder and Chair of the GRSS German Chapter (2003-2008), served as Associate Editor for the IEEE GRS Letters (20032007) and for the IEEE TGRS (since 2005). He and his colleagues received the GRSS Transactions Prize Paper Awards in 1997, 2001, and 2007, the IEEE W.R.G. Baker Award in 2012 from the IEEE Board of Directors and were nominated for the Federal Prize for Innovation and Future Technologies of the German President (2012). He is also recipient of several awards including the DLR Science Award (1995), IEEE Nathanson Award for the Young Radar Engineer of the Year (1999), the IEEE Kiyo Tomiyasu Field Award (2007), and the IEEE GRSS Distinguished Achievement Award (2014). He served as a member of the Board of Directors of the Information Technology Society of the German Association for Electrical, Electronic and Information Technologies (2003-2008), as chair of the Scientific and Technical Council of DLR (2009-2011), and as chair of the DLR's Center of Excellence on Synthetic Aperture Radar (2000-2015). He has participated as a member of the technical program committee of more than 50 conferences and served as general chairman for EUSAR'06, as co-chair of the European Radar Conference (EuRAD'07) and as general co-chair for IGARSS'12. Since 2012 he is serving as the Principal Investigator (PI) for the Helmholtz Research Alliance "Remote Sensing and Earth System Dynamics", comprising of 18 research institutes and 30 associated partners. 


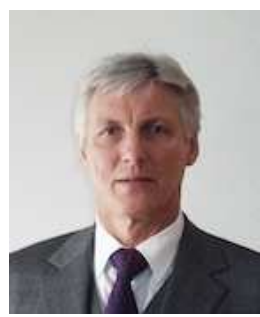

Werner Wiesbeck (SM 87, F 94) received the Dipl.-Ing. (M.S.E.E.) and the Dr.-Ing. (Ph.D.E.E.) degrees from the Technical University Munich in 1969 and 1972, respectively. From 1972 to 1983 he was with AEG-Telefunken in various positions including that of head of R\&D of the Microwave Division in Flensburg and marketing director Receiver and Direction Finder Division, Ulm. During this period he had product responsibility for mm-wave radars, receivers, direction finders and electronic warfare systems. From 1983 to 2007 he was the Director of the Institut für Höchstfrequenztechnik und Elektronik (IHE) at the University of Karlsruhe (TH) and he is now Distinguished Senior Fellow at the Karlsruhe Institute of Technology (KIT). Research topics include antennas, wave propagation, Radar, remote sensing, wireless communication and Ultra Wideband technologies. He is author and co-author of several books and of more than 800 publications, supervisor of more than $90 \mathrm{PhDs}$, and responsible supervisor of over 600 Diploma-/Master theses and he holds over 60 patents. In 1989 and 1994, respectively, he spent a six months sabbatical at the Jet Propulsion Laboratory, Pasadena. He is a member of the IEEE GRS-S AdCom (1992-2003), Chairman of the GRS-S Awards Committee (1994 - 1998, 2002 - ), Executive Vice President IEEE GRS-S (1998-1999), President IEEE GRSS (2000-2001), Associate Editor IEEE-AP Transactions (1996-1999), past Treasurer of the IEEE German Section (1987-1996, 2003-2007). He has been General Chairman of the ' 88 Heinrich Hertz Centennial Symposium, the '93 Conference on Microwaves and Optics (MIOP '93), the Technical Chairman of International mm-Wave and Infrared Conference 2004, Chairman of the German Microwave Conference GeMIC 2006 and he has been a member of the scientific committees and TPCs of many conferences. For the Carl Cranz Series for Scientific Education he serves as a permanent lecturer for Radar systems engineering, wave propagation and mobile communication network planning. He is a member of an Advisory Committee of the EU - Joint Research Centre (Ispra/Italy), and he is an advisor to the German Research Council (DFG), to the Federal German Ministry for Research (BMBF) and to industry in Germany. $\mathrm{He}$ is the recipient of a number of awards, lately the IEEE Millennium Award, the IEEE GRS Distinguished Achievement Award, the Honorary Doctorate (Dr. h.c.) from the University Budapest/Hungary, the Honorary Doctorate (Dr.-Ing. E.h.) from the University Duisburg/Germany, the Honorary Doctorate (Dr.-Ing. E.h.) from the Technische Universität Ilmenau and the IEEE Electromagnetics Award 2008. He is a Life Fellow of IEEE, an Honorary Life Member of IEEE GRS-S, a Member of the Heidelberger Academy of Sciences and Humanities and a Member of the German Academy of Engineering and Technology (acatech). He is a member of a number of national and international Awards Committees. 